\title{
An informed nation is a healthier nation
}

\section{Editorial}

During this season of the year individuals often make health related commitments to themselves to enhance the quality of their lives. The US Surgeon General Family History Initiative enables the nationwide community to engage in preventive health and become proactive in making informed decisions beneficial to their overall well-being.

The US Surgeon General Family History Initiative Web tool can be utilized by individuals of the general public to record familial transmission of various healthy as well as pathological genes of their maternal and paternal biological relatives expanded at least 3 generations. Since some individuals may not personally know their intergenerational biological relatives they must interview other family members and/or friends in order to obtain accurate information. Karyo types of all human beings contain healthy as well as pathological genes related to the onset of diseases. The presence of a pathological gene does not automatically lead to the disease manifestation affiliated with it; multiple factors are associated with the onset of disease.

The US Surgeon General Family History Initiative enables the nationwide community to view the onset of preventable chronic disorders such as obesity and heart disease manifesting among biological relatives from one generation to the next. The repetitive manifestation of intergenerational diseases among biological relatives is not only due to the presence of pathological genes but it is also influence by the presence of particular social determinants of health such as the level of the socioeconomic status and educational attainment, type of behavioral habits, social support, and environmental conditions, and access to health care. Multifactorial influences such as cigarette smoking, substance abuse, sedentary lifestyles, chronic high fatty diets, chronic high stress level, hazardous environmental conditions involving air and/or water pollution, low socioeconomic status, low educational attainment, and inadequate access to health care lead to the heightened manifestation of preventable chronic diseases causing health disparities to flourish among certain populations of people.

If individuals are aware of the composite traits present in their genomes, then they will be empowered to make educated decisions regarding their health, for example: Although individuals may possess the gene for diabetes mellitus, they may take the necessary precautions to prevent the expression of this trait such as adhering to a nutritious diet, obtaining adequate exercise, maintaining weight control, having a strong social support system, and experiencing lower levels of stress. By contrast, if individuals are unaware of their family medical histories, then they are unable to make accurate and informed decisions regarding their health.

Genetic testing should be performed for individuals whose family medical histories reveal the intergenerational manifestation of unavoidable diseases, for example: Breast cancer demonstrating an intergenerational transmission among women of a family may lead
Volume 3 Issue 2 - 2015

\author{
Apryl Brown \\ Department of Science, MPH,Wayne County Community \\ College District, USA
}

Correspondence: Apryl Brown, Department of Science, Executive Committee Member-at-Large Officer of Delta Omega Honorary Society in Public Health Founder of the WCCCD Xi Alpha TriBeta Club affiliated with the Beta Beta National Biological Honor Society P.O. Box 442047 Detroit, MI 48244 USA,Email abrown2@wcccd.edu

Received: December 21, 2015 | Published: December 22, 2015

to them being tested for the presence of the BRCA gene which is affiliated with this disease.

Individuals possessing well investigated family medical histories are more capable of providing accurate medical documentation to their health care providers when medical attention is being rendered to them. Information derived from well documented family medical histories enables health care providers to make more precise differential diagnoses when treating their patients.

According to the US Surgeon General Family History Initiative, although $96 \%$ of Americans understand the importance of obtaining their family medical histories only $1 / 3$ of these individuals have ever recorded this document. It is imperative for family relatives to become informed and relay their medical histories to one another. For that reason, the US Surgeon General declared Thanksgiving as the National Family History Day, since during this holiday period family members potentially come together and share information pertaining to medical histories that are seen within their biological relatives born in various generations.

Advocating for the nationwide community to know their family medical histories will allow people to actively engage in pertinent decisions pertaining to their health including disease prevention, early disease detection, and health promotion. This endeavor would help decrease the community epidemic manifestation of preventable pathological conditions occurring in various regions seen throughout the nation.

\section{Acknowledgements}

None.

\section{Conflict of interest}

The author declares no conflict of interest. 